Adedunni Wumi Olusanya ${ }^{1}$, Oluwarotimi Bolaji Olopade², Ifedayo Adeola Odeniyi ${ }^{2,} 3$, Olufemi Adetola Fasanmade2, 3

${ }^{1}$ Department of Pharmacology, Therapeutics and Toxicology, College of Medicine, University of Lagos, Idi-Araba Lagos, Nigeria

2Department of Medicine, Lagos University Teaching Hospital, Idi-Araba Lagos, Nigeria

${ }^{3}$ Department of Medicine, College of Medicine, University of Lagos, Idi-Araba Lagos, Nigeria

\title{
Patterns of drug use among type 2 diabetic patients with comorbidities attending a tertiary centre in Lagos, Nigeria
}

\section{ABSTRACT}

Background. Diabetes care involves the use of drugs to control hyperglycaemia and the treatment of comorbid disorders to prevent cardiovascular morbidity and other complications. This study investigated patterns of comorbidities and drug use among diabetic patients at a tertiary centre in Lagos, Nigeria.

Methods. This was a cross-sectional study carried out among 216 patients with type 2 DM attending the Diabetes Clinic of a tertiary centre. Data was captured by using a questionnaire that documented biodata, number of comorbidities, number and combination of drugs for each participant and analysed using SPSS version 18.

Results. Comorbidities were present among 215 out of 216 (99.54\%) participants and hypertension and dyslipidaemia were the most common comorbid disorders. The number of pills consumed per patient ranged from 1 to 10 with a mean of $4.78 \pm 1.73$ and $57.4 \%$ were on 5 pills or more. A fixed-dose combination was used in $37(17.1 \%)$ of the patients. The majority of the patients were on metformin as monotherapy or in combination therapy. Antihypertensive most prescribed were renin-angiotensin system (RAS) blockers which were

Address for correspondence: Adedunni Wumi Olusanya

Department of Pharmacology

Therapeutics and Toxicology

College of Medicine, University of Lagos, Lagos, Nigeria

e-mail dedunolusanya@gmail.com,dedunolusanya@unilag.edu.ng

Clinical Diabetology 2021, 10; 4: 342-348

DOI: 10.5603/DK.a2021.0043

Received: 30.09 .2020

Accepted: 28.12.2020 prescribed in $73 \%$ of participants. Antiplatelet drugs were used for both primary and secondary prevention of CVDs. Statin was used in less than $50 \%$ of the population. Double RAS blockade was also observed in $6 \%$ of participants.

Conclusions. Comorbidities were common in the diabetic population. Pill load was high but appropriate in most patients. Statin uptake was suboptimal while there was increased uptake of antiplatelet drugs among participants. (Clin Diabetol 2021; 10; 4: 342-348)

Key words: diabetes mellitus, comorbidities, antidiabetic drugs, type 2 DM, antihypertensive drugs

\section{Introduction}

Type 2 Diabetes mellitus (T2DM) is one of the most common cardiovascular risk factors and is associated with high morbidity and mortality globally. It is a chronic disease characterized by relative or/and absolute insulin deficiency and insulin insensitivity which results in deficient metabolism of carbohydrate, protein, and lipids [1]. International Diabetes Federation (IDF) 2019 reported Diabetes prevalence in Nigeria as $3 \%$, but this is probably underestimated because a systematic review by Uloko and colleagues published in 2018 found the prevalence of DM in Nigeria to be $5.77 \%[2,3]$.

The complications arising from diabetes mellitus (DM) are major contributors to increased hospitalization and mortality. Complications from DM include atherosclerotic cardiovascular diseases such as coronary 
heart disease, cerebrovascular disease, or peripheral arterial disease (PAD) presumed to be of atherosclerotic origin and microvascular complications such as chronic kidney disease, retinopathy, and neuropathy [4]. Appropriate management of DM helps to prevent these complications.

The management of DM addresses glycaemic control and the prevention and management of complications. It also involves the treatment of comorbid cardiovascular risk factors like hypertension, dyslipidaemia, obesity $[4,5]$. Consequently, patients with DM often require a relatively high number of drugs to achieve therapeutic goals.

The high pill load among this population calls for caution because of the increased risk of drug-drug interactions and drug-disease interaction which may lead to reduced drug efficacy or adverse drug effects especially in the elderly [6]. This is a result of declining organ function and multiple comorbid conditions that occurs with ageing. Furthermore, the prevalence of DM is disproportionately higher in the older population because of increasing life expectancy especially in developing countries and this emphasises the need to address drug use patterns among individuals with diabetes. Increased mortality in hospitalised elderly patients has been associated with drug reactions [7]. Therefore, the use of appropriate drug therapy is imperative in diabetic care.

Evidence-based guidelines have been developed to optimize treatment outcome and improve the quality of care among patients with DM. These guidelines provide recommendations for managing hyperglycaemia as well as the management of comorbidities and complications. Guidelines used in DM care include publications from the International Diabetes Federation (IDF), the American Diabetes Association (ADA), the American Association of Clinical Endocrinologists (AACE) and the Diabetes Association of Nigeria (DAN) $[2,4,5,8]$. Studies have shown gaps in implementing guidelines in the management of patients with chronic diseases $[9,10]$.

Most studies have evaluated the use of glucoselowering drugs among persons with DM, with little attention paid to the management of comorbid conditions among this population. Poor management of comorbidities and inappropriate drug combinations may obliterate the gains from glycaemic control. Therefore, it is important to evaluate the presence and treatment of comorbidities to improve clinical outcome in Diabetic care. This study investigated comorbidities and assessed the pattern of drug use concerning the presence of comorbid disorders in out-patient Type 2 diabetic patients.

\section{Material and methods}

Study site

The study was conducted at the diabetes outpatient clinic of the Department of Medicine, Lagos University Teaching Hospital (LUTH).

\section{Study design}

This was a cross-sectional study carried out among patients with type 2 DM for three months after obtaining approval from the LUTH Health Research Ethics committee. A total of 216 patients who consented were included in the study.

Inclusion criteria were men and women aged 18 years and above with a diagnosis of type 2 diabetes mellitus (T2DM). Exclusion criteria are Type 1 diabetic and gestational diabetes. Questionnaires were administered by Medical House officers, who were blinded to the aims of the study. Patients were interviewed using a standard questionnaire and case notes were used to corroborate medications orders. Data was collected for demographic details (names, age, and sex of patients), diagnosis and comorbidities. The comorbidities assessed in this study include hypertension, dyslipidaemia, obesity, diabetes mellitus foot syndrome (DMFS), chronic kidney disease (CKD) and stroke. These comorbidities were ascertained based on patients' report or a diagnosis of the specific comorbidity in the case note. The prescribed drug, including the branded and generic name of all drugs used by patients (both prescription and non-prescription drugs), dose, dose frequency and route of administration were also recorded. Data were analysed using IBM SPSS version -18. Data were presented as frequencies, percentages, mean and standard deviation.

\section{Results}

A total of 216 patients between the ages of 34 to 89 years were recruited for the study. The duration of DM ranged from 1-46 years. At least one comorbidity was present in $99.54 \%$ of the population. Other demographic and comorbidity patterns are shown in Table 1.

\section{Patterns of drug use}

The number of pills consumed per patient was from 1 to 10 with a mean of $4.78 \pm 1.73$ (Fig.1). The majority (57.4\%) were on 5 pills or more. A fixed-dose combination was used in $37(17.1 \%)$ of the patients.

Drugs commonly used are represented in figure 2 below. Antidiabetic was used by 215 out of 216 diabetic patients, one patient was on dietary therapy. A total of $167(77.31 \%)$ patients were on antihypertensive drugs against $153(70.8 \%)$ patients who reported hypertension. Statins were utilized in $116(53.7 \%)$ of the 


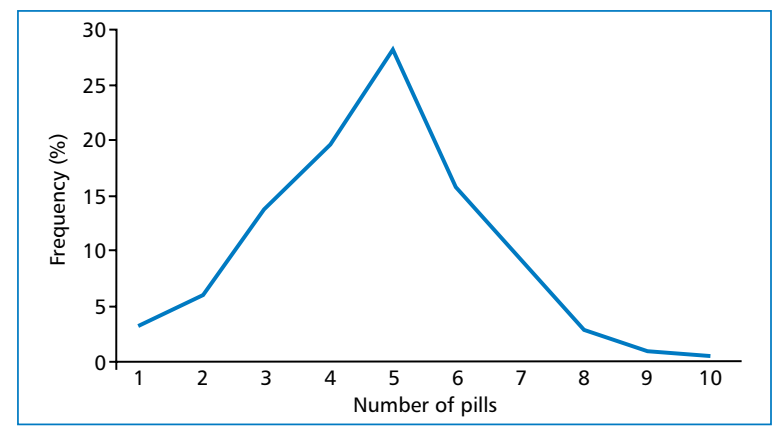

Figure 1. Pill count among participants

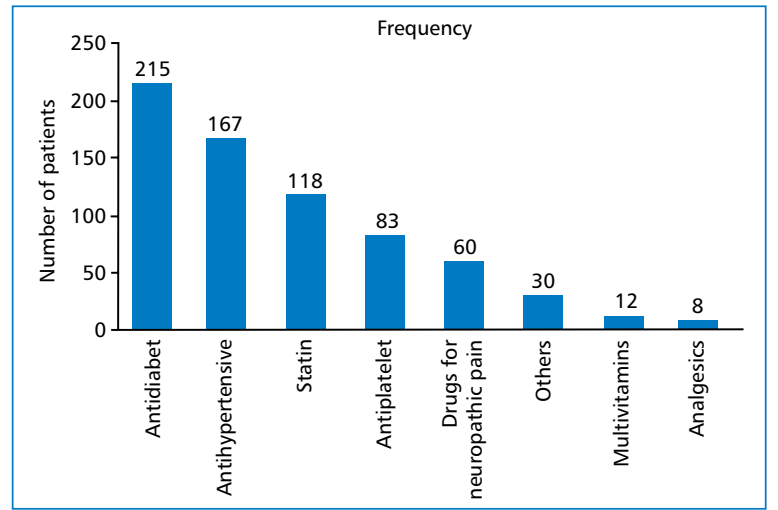

Figure 2. Classes of drug use among participants

participants whereas a total of 106 (49.1\%) patients reported dyslipidaemia.

Other drugs such as antacids, sildenafil, antiviral drugs, proton pump inhibitors, tamsulosin, antifungal, steroids and antiandrogen were used in less than $14 \%$. Analgesics include tramadol, didydrocodeine and diclofenac, celecoxib. This is shown in figure 1.

Sulphonylureas (SU) used were long-acting glibenclamide, and glimepiride and short-acting gliclazide. Dipeptidyl peptidase-4 (DPP4) inhibitors included vildagliptin, sitagliptin, linagliptin. Glucagon-like peptide-1 (GLP-1) receptor agonists used was liraglutide while the only thiazolidinedione used was pioglitazone (See supplementary Table 1).

An inappropriate combination of two drugs from the sulphonylurea class was observed in 3 patients and double RAS blockade was also observed in about 13 (5\%) patients (see supplementary Tables 1 and 2).

\section{Discussion}

The study represented a population of type 2 diabetes mellitus in a tertiary health centre, the majority of the patients were 40 years and above and comorbidities
Table 1. Demography and comorbidity patterns

\begin{tabular}{|c|c|c|}
\hline Parameter & $n$ & $\%$ \\
\hline \multicolumn{3}{|l|}{ Age (years) } \\
\hline $34-39$ & 6 & 2.8 \\
\hline $40-59$ & 157 & 72.7 \\
\hline 60 and above & 53 & 24.5 \\
\hline \multicolumn{3}{|l|}{ Gender } \\
\hline Male & 87 & 40.3 \\
\hline Female & 129 & 59.7 \\
\hline \multicolumn{3}{|l|}{ Level of education } \\
\hline None & 11 & 5.1 \\
\hline Primary & 37 & 17.1 \\
\hline Secondary & 69 & 31.9 \\
\hline Tertiary & 99 & 45.9 \\
\hline \multicolumn{3}{|c|}{$\begin{array}{l}\text { Duration of diabetes mellitus in } \\
\text { years }\end{array}$} \\
\hline Less than 10 & 104 & 48.1 \\
\hline $10-19$ & 78 & 26.2 \\
\hline 20 and above & 34 & 15.7 \\
\hline \multicolumn{3}{|c|}{ Number of comorbidities } \\
\hline 0 & 1 & 0.46 \\
\hline 1 & 1 & 0.46 \\
\hline 2 & 7 & 3.24 \\
\hline 3 & 56 & 25.93 \\
\hline 4 & 91 & 42.13 \\
\hline 5 & 60 & 27.78 \\
\hline \multicolumn{3}{|l|}{ Comorbidities } \\
\hline Hypertension & 153 & 70.8 \\
\hline Chronic kidney disease & 15 & 6.9 \\
\hline Stroke & 17 & 7.9 \\
\hline Obesity & 68 & 31.5 \\
\hline Dyslipidaemia & 106 & 49.1 \\
\hline DMFS & 27 & 12.5 \\
\hline
\end{tabular}

were common. Hypertension and dyslipidaemia were the most common comorbid conditions seen in the population. This study findings are similar to that of a multicentre study in sub-Saharan Africa which reported high rates of comorbid conditions, hypertension was the most common which was reported in $71 \%$ of the population. This was followed by dyslipidaemia which was observed in 34\% [11].

The study shows that the pill load among participants was quite high, the majority $(57.4 \%)$ of the participants were on 5 or more pills. This agrees with previous reports of a high drug burden among indi- 
Table 2. Antidiabetic drug use among study participants

\begin{tabular}{lcc}
\hline Drug Class & $\mathbf{n}$ & $\%$ \\
\hline Biguanide (Metformin) & 183 & 84.7 \\
Sulphonylurea & 113 & 52.3 \\
Glibenclamide & 8 & 3.6 \\
Glimepiride & 70 & 31.8 \\
Gliclazide & 35 & 15.9 \\
Insulin analogues & 54 & 25 \\
DPP4I & 47 & 21.8 \\
Thiazolidinediones & 7 & 3.2 \\
GLP-1 receptor analogue & 2 & 0.9 \\
No of drugs & & \\
One & 63 & 29.0 \\
Two & 106 & 48.8 \\
Three & 31 & 14.1 \\
Four & 2 & 0.9 \\
\hline
\end{tabular}

DPP4I: dipeptidyl peptidase-4 inhibitors; SU: sulphonylureas; GLP: glucagon-like peptide-1 (GLP-1) receptor agonists

viduals with T2DM [12-14]. The use of a high number of drugs has been associated with the presence of multi-morbidities among diabetes patients [15]. Multi-morbidities in DM may occur from the presence of other co-morbid cardiovascular risk factors and/or complications of DM.

Generally, the concomitant use of several drugs in a patient which is termed polypharmacy is discouraged because of the increased risk of drug-related problems such as poor drug adherence, drug-drug interaction, drug-disease interaction, adverse effects, and increased cost of medications [16]. Most studies have used an arbitrary number of five drugs to define polypharmacy and have described the use of five or more drugs as irrational drug use. While the fear of polypharmacy is rational, it has been recommended that rational drug use should be judged based on the appropriateness of therapy rather than an absolute pill count [17].

Although the population studied are on polypharmacy, the drug use pattern appears to be appropriate for the majority of the patients in this study. The use of fewer than five drugs in this population may result in under-treatment because the majority of the patients had co-morbid diseases.

The classes of drugs used in this study correlate with the patterns of co-morbidity seen in the population (Fig. 1). The most commonly prescribed antidiabetic drug in the study was metformin, which was used in two-thirds of the patients on monotherapy and in over $90 \%$ of the patients on polytherapy (Table 3 ). This
Table 3. Antihypertensive use pattern among participants

\begin{tabular}{lcc}
\hline Drug Class & $\mathbf{n}$ & $\%$ \\
\hline CCB & 87 & 40.3 \\
ACEI & 78 & 36.1 \\
ARB & 58 & 26.9 \\
Diuretics & 68 & 31.5 \\
BB & 25 & 11.6 \\
ALPHA AGO & 2 & 0.9 \\
No of drugs & & \\
One & 79 & 36.6 \\
Two & 59 & 27.3 \\
Three & 27 & 12.5 \\
Four & 10 & 4.6 \\
Seven & 1 & 0.5 \\
\hline
\end{tabular}

CCB: calcium channel blockers; ACEI: angiotensin-converting enzyme inhibitors; ARB: angiotensin receptor blockers; BB: beta-blockers; ALPHA AGO: and centrally acting alpha agonists. Two diuretics include combinations of indapamide + frusemide, hydrochlorthiazide + frusemide, spironolactone + torsemide and hydrochlorthiazide + spironolactone

is in line with the DAN, AACE/ACE and ADA guidelines that recommend the use of metformin as the first-line drug and as add-on therapy in those who need a second antidiabetic drug in type $2 \mathrm{DM}$ provided there is no contraindication to the use of metformin $[4,5,8]$. Metformin is effective in lowering HBA1C, weight and showed reduced CVS mortality compared to SU [18].

Other studies have also reported a preference for metformin as a first-line drug and also used frequently in combination therapy [19]. The use of DPP4I and alpha-glucosidase inhibitors as preferred first-line drugs have been documented in other regions for instance a study reported the use of alpha-glucosidase inhibitors in Beijing, China [20].

SUs were the second most prescribed drugs either in monotherapy or in combination with other drugs. This pattern has been described in other studies in Nigeria [21]. The most used SU in this study was glimepiride with a relatively shorter half-life compared to glibenclamide. This conflicts with previous reports of a high uptake of glibenclamide which has a long halflife [22]. SU with shorter half-lives are associated with a lower incidence of hypoglycaemia and are therefore preferred especially in the elderly who are more prone to hypoglycaemia.

Other drug classes used among participants in this study include insulin analogues in 25\%, DPP4I in $21.8 \%$, GLP-1 agonist in $0.5 \%$, and thiazolidinedione in $0.5 \%$. ADA guidelines recommend the early introduction of insulin in patients with glycated $\mathrm{HB}$ above 
Table 4. Statin and antiplatelet use among participants

\begin{tabular}{lcc}
\hline Drug & $\mathbf{n}$ & $\%$ \\
\hline Statin use & 118 & $\mathbf{5 4 . 6}$ \\
High-Intensity Statin & 14 & 6.5 \\
Medium Intensity Statin & 92 & 42.6 \\
Low-Intensity Statin & 2 & 0.9 \\
Antiplatelet & 82 & 38 \\
Clopidogrel & 11 & 5.1 \\
Aspirin & 71 & 32.9 \\
\hline
\end{tabular}

Statins used include atorvastatin, rosuvastatin and simvastatin

$10 \%$ to reduce cardiovascular morbidity [4] AACE on the other hand recommended the addition of insulin when $\mathrm{HbA} 1 \mathrm{c}$ is above $9 \%$ with symptoms [5]. The high rate of insulin use observed in this study may reflect higher baseline HBA1c or more advanced B-islet cell failure in the population. An increasing trend towards the use of DPP4I was also observed and may be due to its lower risk of hypoglycaemia and weight naïve effect compared to SU [21, 22].

Overall, antidiabetic drug use in this population complies with available guidelines, although the prescription was limited to four classes of antidiabetic drugs. This may be related to tolerability, cost, availability, and physician's preference. Consideration for drugs classes like SGLT is important because of its benefit in patients with heart failure [4].

Antihypertensive drugs were used in $79.2 \%$ of the population. This is disproportionately higher compared to the number who had hypertension. The higher rates may be attributed to the use of these drugs in other conditions, for example, ACEI or ARB are used in the management of proteinuria and beta-blockers in arrhythmias. Drugs used in monotherapy include calcium channel blockers (CCB), angiotensin-converting enzyme inhibitors ( $A C E I$ ), angiotensin receptor blockers (ARB), diuretics and beta-blockers (BB). The use of CCB and thiazide diuretics as initial monotherapy follows the JNC 8 guideline which recommends the use of CCB and thiazide diuretics as first-line agents in black diabetic patients with co-morbid hypertension. This is because these classes of drugs have been associated with a more favourable cardiovascular outcome when compared to other classes of hypertensive drugs among blacks with DM [22]. Other guidelines have however recommended the use of ACEI or ARB as the preferred first-line in diabetics because of the protective effect on nephropathy and retinopathy $[4,5,8]$. A large proportion of the participants are on either ACEI or ARB. Further comparative study on antihypertensive drugs is needed to provide robust evidence guiding the use of the first-line antihypertensive drug in blacks with DM.

An inappropriate combination of antihypertensive was observed in the participants and was associated with polypills. About $6 \%$ were on double RAS blockade. Initially, dual angiotensin blockade was advocated because the combination was shown to improve proteinuria compared to a single agent from either class but recent evidence from randomised controlled trials showed higher risks of adverse effects like hyperkalaemia and declining renal function [24, 25]. As a result, the use of double RAS blockade in hypertension is no longer recommended [24]. A regular review of the medication is important to correct such practices even though many of these patients attend multiple clinics (nephrology, cardiology, and neurology) due to their co-morbid states.

Statins are indicated for the treatment of dyslipidaemia and the prevention of macrovascular complications in patients with DM $[4,5,8]$. The ADA recommends the use of statin in all patients between 40 - 75 years irrespective of CVS risk and levels of cholesterol whereas statin use is recommended in the other guidelines based on risk stratification and cholesterol goals $[5,8]$. There is a uniform consensus on the use of high-intensity statins for secondary prevention of atherosclerotic cardiovascular disease (ASCVD).

In this study, only $54.6 \%$ of the participants were on statin, of these only $6.5 \%$ were on a high-intensity statin. This appears low considering that $49.1 \%$ had dyslipidaemia, $7.9 \%$ had a stroke and $12.5 \%$ had diabetes mellitus foot syndrome. DMFS may also suggest ASCVD because the condition occurs secondary to atherosclerotic peripheral vascular disease, peripheral neuropathy and infections. Presented findings suggest that statins were prescribed in patients who had dyslipidaemia and ASCVD. Comparing with the ADA guideline, statin uptake is low since more than $95 \%$ of the population were 40 years and above. Low statin uptake has been reported in other studies, a study evaluating statin use among high-risk patients showed only about half of the patients $47.5 \%$ were on statin therapy [26].

Antiplatelet therapy using aspirin at a dose of 75-162 mg/day is recommended for the secondary prevention of ASCVD in DM patients with a history of atherosclerotic cardiovascular disease [4]. Clopidogrel is recommended in patients with aspirin allergy [4]. There is no uniform consensus on the use of antiplatelet in primary ASCVD prevention. Antiplatelet drugs were used in $38 \%$ of the population and this is disproportionally high compared to ASCVD prevalence. The number of patients on antiplatelet exceeds the number of 
participants with stroke and DMFS. This suggests that antiplatelet were used in both secondary and primary prevention of ASCVD. Risk stratification and discussion with the patient is advised or essential if aspirin is considered for primary prevention [4].

Pregabalin and gabapentin were used in about a tenth of the population for the treatment of neuropathic pain. The pattern of drug treatment largely complies with the ADA guideline which recommends the use of pregabalin, gabapentin and duloxetine for treating neuropathic pain in DM (ADA) [4]. A few patients were on nutritional supplements, supplements are not recommended unless there are specific indications [4].

Although several studies have been carried out on drug use in diabetes, most of these only documented the use of antidiabetic or antihypertensive drugs, to the best of the authors' knowledge, this study is one of the few studies that investigated all drug classes used in DM patients. It has been identified that a high drug load was appropriate in the majority of the patients.

\section{Conclusion}

This study reported comorbidities and drug use pattern among diabetic patients and a tendency to use a large number of drugs which is appropriate because of the presence of co-morbid conditions. It also identified inappropriate drug combination patterns. There is a need to continue regular review of medications to optimize patient care.

\section{Conflict of interest}

The authors report no competing interests.

\section{REFERENCES}

1. Skyler JS, Bakris GL, Bonifacio E, et al. Differentiation of Diabetes by Pathophysiology, Natural History, and Prognosis. Diabetes. 2017; 66(2): 241-255, doi: 10.2337/db16-0806, indexed in Pubmed: 27980006.

2. Diabetes atlas 9th ed. International Diabetes Federation. Brussels. 2019.

3. Uloko $A E$, Musa BM, Ramalan MA, et al. Prevalence and Risk Factors for Diabetes Mellitus in Nigeria: A Systematic Review and Meta-Analysis. Diabetes Ther. 2018; 9(3): 1307-1316, doi: 10.1007/s13300-018-0441-1, indexed in Pubmed: 29761289.

4. American Diabetes Association. 9. Pharmacologic Approaches to Glycemic Treatment: . Diabetes Care. 2019; 42(Suppl 1): S90S9S102, doi: 10.2337/dc19-S009, indexed in Pubmed: 30559235.

5. Garber AJ, Abrahamson MJ, Barzilay Jl, et al. Consensus statement by the American Association of Clinical Endocrinologists and American College of Endocrinology on The Comprehensive Type 2 Diabetes Management Algorithm - 2019 Executive Summary. Endocr Pract. 2019; 25(1): 69-100, doi: 10.4158/CS-2018-0535, indexed in Pubmed: 30742570.

6. Peron EP, Ogbonna KC, Donohoe KL. Antidiabetic medications and polypharmacy. Clin Geriatr Med. 2015; 31(1): 17-27, vii, doi: 10.1016/j.cger.2014.08.017, indexed in Pubmed: 25453298.

7. Patel TK, Patel PB. Mortality among patients due to adverse drug reactions that lead to hospitalization: a meta-analysis. Eur J Clin
Pharmacol. 2018; 74(6): 819-832, doi: 10.1007/s00228-0182441-5, indexed in Pubmed: 29556685.

8. Clinical Practice Guidelines for Diabetes Management in Nigeria Diabetes Association of Nigeria. Nigeria 2013.

9. Engel J, Damen NL, van der Wulp I, et al. Adherence to Cardiac Practice Guidelines in the Management of Non-ST-Elevation Acute Coronary Syndromes: A Systematic Literature Review. Curr Cardiol Rev. 2017; 13(1): 3-27, doi: 10.2174/1573403×1266616050410 0025, indexed in Pubmed: 27142050.

10. Hageman D, Pesser N, Gommans LNM, et al. Limited Adherence to Peripheral Arterial Disease Guidelines and Suboptimal Ankle Brachial Index Reliability in Dutch Primary Care. Eur J Vasc Endovasc Surg. 2018; 55(6): 867-873, doi: 10.1016/j.ejvs.2018.02.011, indexed in Pubmed: 29576335.

11. Ekoru K, Doumatey A, Bentley AR, et al. Type 2 diabetes complications and comorbidity in Sub-Saharan Africans. EClinicalMedicine. 2019; 16: 30-41, doi: 10.1016/j.eclinm.2019.09.001, indexed in Pubmed: 31832618.

12. Alwhaibi M, Balkhi B, Alhawassi TM, et al. Polypharmacy among patients with diabetes: a cross-sectional retrospective study in a tertiary hospital in Saudi Arabia. BMJ Open. 2018; 8(5): e020852, doi: 10.1136/bmjopen-2017-020852, indexed in Pubmed: 29794097.

13. Noale M, Veronese N, Cavallo Perin P, et al. Polypharmacy in elderly patients with type 2 diabetes receiving oral antidiabetic treatment. Acta Diabetol. 2016; 53(2): 323-330, doi: 10.1007/ s00592-015-0790-4, indexed in Pubmed: 26155958.

14. Adisa R, Fakeye TO. Effect of number and type of antidiabetes medications on adherence and glycemia of ambulatory type 2 diabetes patients in southwestern Nigeria. Pharm Pract (Granada). 2013; 11(3): 156-165, doi: 10.4321/s1886-36552013000300006, indexed in Pubmed: 24223081.

15. Geitona M, Latsou D, Toska A, et al. Polypharmacy and Adherence Among Diabetic Patients in Greece. Consult Pharm. 2018; 33(10): 562-571, doi: 10.4140/TCP.n.2018.562., indexed in Pubmed: 30322433.

16. Balkhi B, AlQahtani N, Alwhaibi M, et al. Prevalence and Factors Associated With Polypharmacy Use Among Adult Patients in Saudi Arabia. J Patient Saf. 2017 [Epub ahead of print], doi: 10.1097/ PTS.0000000000000439, indexed in Pubmed: 29087978.

17. World Health Organization (WHO). World Health Organization Technical Report; Medication Safety in Polypharmacy World Health Organization, Geneva, Switzerland; 2019.

18. Bolen S, Tseng E, Hutfless S, et al. Diabetes Medications for Adults With Type 2 Diabetes: An Update. Rockville (MD): Agency for Healthcare Research and Quality (US); 2016. indexed in PubMed: 27227214.

19. Moreno Juste A, Menditto E, Orlando V, et al. Treatment Patterns of Diabetes in Italy: A Population-Based Study. Front Pharmacol. 2019; 10: 870, doi: 10.3389/fphar.2019.00870, indexed in Pubmed: 31447672 .

20. Nishimura R, Kato H, Kisanuki K, et al. Treatment patterns, persistence and adherence rates in patients with type 2 diabetes mellitus in Japan: a claims-based cohort study. BMJ Open. 2019; 9(3): e025806, doi: 10.1136/bmjopen-2018-025806, indexed in Pubmed: 30826768.

21. Fadare J, Olamoyegun M, Gbadegesin BA. Medication adherence and direct treatment cost among diabetes patients attending a tertiary healthcare facility in Ogbomosho, Nigeria. Malawi Med J. $2015 ; 27(2)$ : 65-70, doi: $10.4314 / \mathrm{mmj} . v 27 i 2.7$, indexed in Pubmed: 26405515.

22. Deacon CF, Lebovitz HE. Comparative review of dipeptidyl peptidase-4 inhibitors and sulphonylureas. Diabetes Obes Metab. 2016; 18(4): 333-347, doi: 10.1111/dom.12610, indexed in Pubmed: 26597596.

23. James PA, Oparil S, Carter BL, et al. 2014 evidence-based guideline for the management of high blood pressure in adults: report from the panel members appointed to the Eighth Joint National 
Committee (JNC 8). JAMA. 2014; 311(5): 507-520, doi: 10.1001/ jama.2013.284427, indexed in Pubmed: 24352797.

24. Mogensen CE, Neldam S, Tikkanen I, et al. Randomised controlled trial of dual blockade of renin-angiotensin system in patients with hypertension, microalbuminuria, and non-insulin dependent diabetes: the candesartan and lisinopril microalbuminuria (CALM) study. BMJ. 2000; 321(7274): 1440-1444, doi: 10.1136/ bmj.321.7274.1440, indexed in Pubmed: 11110735.
25. Jennings DL, Kalus JS, Coleman Cl, et al. Combination therapy with an ACE inhibitor and an angiotensin receptor blocker for diabetic nephropathy: a meta-analysis. Diabet Med. 2007; 24(5): 486-493, doi: 10.1111/j.1464-5491.2007.02097.x, indexed in Pubmed: 17367311.

26. Ferrières J, Gorcyca K, lorga ŞR, et al. Lipid-lowering Therapy and Goal Achievement in High-risk Patients From French General Practice. Clin Ther. 2018; 40(9): 1484-1495.e22, doi: 10.1016/j. clinthera.2018.07.008, indexed in Pubmed: 30126705. 